\title{
Experimental investigation of vortex properties in a turbulent boundary layer
}

\author{
Bharathram Ganapathisubramani, ${ }^{\text {a) }}$ Ellen K. Longmire, and Ivan Marusic \\ Department of Aerospace Engineering and Mechanics, University of Minnesota, 107 Akerman Hall, \\ 110 Union Street SE, Minneapolis, Minnesota 55455
}

(Received 4 October 2005; accepted 11 March 2006; published online 22 May 2006)

\begin{abstract}
Dual-plane particle image velocimetry experiments were performed in a turbulent boundary layer with $\operatorname{Re}_{\tau}=1160$ to obtain all components of the velocity gradient tensor. Wall-normal locations in the logarithmic and wake region were examined. The availability of the complete gradient tensor facilitates improved identification of vortex cores and determination of their orientation and size. Inclination angles of vortex cores were computed using statistical tools such as two-point correlations and joint probability density functions. Also, a vortex identification technique was employed to identify individual cores and to compute inclination angles directly from instantaneous fields. The results reveal broad distributions of inclination angles at both locations. The results are consistent with the presence of many hairpin vortices which are most frequently inclined downstream at an angle of $45^{\circ}$ with the wall. According to the probability density functions, a relatively small percentage of cores are inclined upstream. The number density of forward leaning cores decreases from the logarithmic to the outer region while the number density of backward-leaning cores remains relatively constant. These trends, together with the correlation statistics, suggest that the backward-leaning cores are part of smaller, weaker structures that have been distorted and convected by larger, predominantly forward-leaning eddies associated with the local shear. () 2006 American Institute of Physics. [DOI: 10.1063/1.2196089]
\end{abstract}

\section{INTRODUCTION}

Over the past few decades, researchers have worked toward understanding the eddy structure within turbulent boundary layers in order to develop effective simplifying models. Adrian, Meinhart, and Tomkins ${ }^{1}$ have reinforced the viewpoint that "hairpin vortices" (based on the hairpin vortex first proposed by Theodorsen ${ }^{2}$ and refined later in various studies $^{3}$ ) are a primary feature in turbulence transport and production. The authors performed particle image velocimetry (PIV) experiments in streamwise-wall-normal planes of a turbulent boundary layer and found signatures of heads of hairpin vortices. Most significantly, they also observed that these vortices traveled together in groups, termed "hairpin packets." Recently, Ganapathisubramani, Longmire, and Marusic, ${ }^{4}$ with stereoscopic PIV data in streamwisespanwise planes, concluded that these hairpin packets occupy only a relatively small percentage of the total area, but contribute to a significant proportion of the total Reynolds shear stress generated, conservatively more than $30 \%$. Hence, the hairpin packets are a very important mechanism in turbulence production. However, a detailed understanding of the three-dimensional structure of hairpin-type vortices and packets is not yet available, and many questions remain unanswered regarding the shape, size, orientation, and dynamics of these eddies.

In the logarithmic region, previous experimental and numerical studies have postulated and documented the exis-

\footnotetext{
${ }^{a)}$ Present address: Center for Aeromechanics Research, University of Texas at Austin, Austin, Texas 78712 .
}

tence of hairpin-like vortices inclined at an angle to the free stream. Theodorsen ${ }^{2}$ postulated the inclined hairpin vortex model to explain the transport of momentum within a boundary layer. Head and Bandyopadhyay ${ }^{5}$ performed flow visualization studies and proposed that the turbulent boundary layer comprised of hairpin/horseshoe type vortices inclined at $45^{\circ}$ to the streamwise direction. Moin and $\mathrm{Kim}^{6}$ and Kim and $\operatorname{Moin}^{7}$ performed correlation studies on vorticity fields in channel flows and concluded that the most probable inclination angle of a vortex is $45^{\circ}$ to the streamwise direction. Experimental studies of structure angle using two-point correlation measurements ${ }^{8}$ have found that the "inferred" angle is seldom equal to $45^{\circ}$. Marusic ${ }^{9}$ performed attached eddy calculations and showed that such a result is consistent with the presence of individual eddies inclined at $45^{\circ}$, given that, however, these $45^{\circ}$ eddies exist over a range of length scales and population densities.

Ong and Wallace ${ }^{10}$ performed a joint probability density analysis with various vorticity components obtained using hot-wire measurements in the near wall and lower edge of the logarithmic region to identify the geometric shape of an average vortex. The authors investigated the $\left(\omega_{x}, \omega_{z}\right)$ covariance (where $\omega_{x}$ is the streamwise vorticity, $\omega_{y}$ is the spanwise vorticity, and $\omega_{z}$ is the wall-normal vorticity) and found that the projection angle in the $x-z$ plane ( $x$ is the streamwise direction, $y$ in the spanwise direction,and $z$ is the wallnormal direction) of the most probable vorticity filament decreases with wall-normal distance from the buffer to the logarithmic region. This angle was computed based on the covariance peaks in the first $\left(\omega_{x}>0\right.$ and $\left.\omega_{z}>0\right)$ and third quadrants $\left(\omega_{x}<0\right.$ and $\left.\omega_{z}<0\right)$. The first and third quadrant 
peaks were chosen to consider the downstream inclined vorticity filaments. However, the covariance plots also revealed the existence of backward-leaning vorticity filaments in the second $\left(\omega_{x}<0\right.$ and $\left.\omega_{z}>0\right)$ and fourth quadrants $\left(\omega_{x}>0\right.$ and $\left.\omega_{z}<0\right)$. The authors also computed the projections on the plan view $x-y$ plane and cross-stream $y-z$ plane; the vorticity filaments that contributed most to the vorticity covariances $\left(\omega_{x}, \omega_{y}\right)$ and $\left(\omega_{y}, \omega_{z}\right)$ had angles of inclination to the $y$ axis that increased with distance from the wall.

Honkan and Andreopoulos ${ }^{11}$ computed the projection angles of the instantaneous vorticity vector (based on multiprobe hot wire measurements) with the $x$ and $z$ axes. They found that for large enstrophy values, the vorticity vector was inclined at $35^{\circ}$ to the streamwise direction ( $x$ axis), and speculated that these orientation angles of the vorticity vector may be related to the presence of hairpin-type vortices.

Almost all previous studies have relied on some global statistical tool to estimate the inclination angle of a vortex. However, the angles inferred from the statistical analysis of velocity and vorticity fields do not necessarily apply to individual vortex cores found in the flow. To resolve the strength and orientation of a vortex, it is necessary not only to measure all three components of vorticity at a point, but also to identify an additional parameter that would isolate vortex cores. Several analytical methods have relied on the complete velocity gradient tensor to isolate individual vortex cores, ${ }^{12,13}$ and Chong, Perry, and Cantwell ${ }^{14}$ have shown that a complete description of the local flow topology can be obtained from the velocity gradient tensor. Recently, Haller ${ }^{15}$ has shown that this description is not complete in flows with significant rotation, however this should not apply to the nominally unidirectional flows considered in this study.

In the present study, a three-camera polarization based dual-plane PIV technique is used to measure the full velocity gradient tensor. The continuity equation is employed in combination with the PIV data to determine the appropriate quantities. The overall objective of this study is to use the three-dimensional velocity gradient data to study the statistical and instantaneous geometric structure of vortex cores in a zero pressure gradient turbulent boundary layer.

\section{EXPERIMENTAL FACILITY AND METHODS}

Experiments were performed in a suction-type boundary layer wind tunnel. Measurement planes were located $3.3 \mathrm{~m}$ downstream of a trip wire in a zero-pressure-gradient flow with freestream velocity $U_{\infty}=5.9 \mathrm{~m} \mathrm{~s}^{-1}$. Hot-wire measurements showed that the turbulence intensity in the free stream was less than $0.2 \%$. The wall shear stress $\left(\tau_{w}\right)$ was computed using the mean velocity profile and the Clauser chart method. The Reynolds number based on boundary layer thickness and skin friction velocity $\mathrm{Re}_{\tau}$ was $1160\left(\mathrm{Re}_{\tau}\right.$ $=\delta U_{\tau} / \nu$, where $\delta$ is the boundary layer thickness, $U_{\tau}$ is the skin friction velocity, and $\nu$ is the kinematic viscosity). The Reynolds number based on the momentum thickness $\operatorname{Re}_{\theta}$ was 2800 and the value of $\delta$ in the region of the measurement planes was $70 \mathrm{~mm}$. The streamwise, spanwise and wall-normal directions are along the $x, y$, and $z$ axes, respectively, and the fluctuating velocity components along those (a)

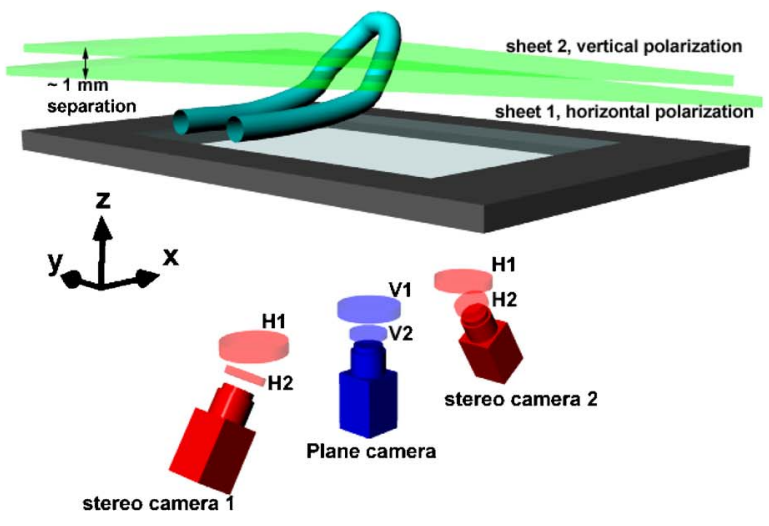

(b)
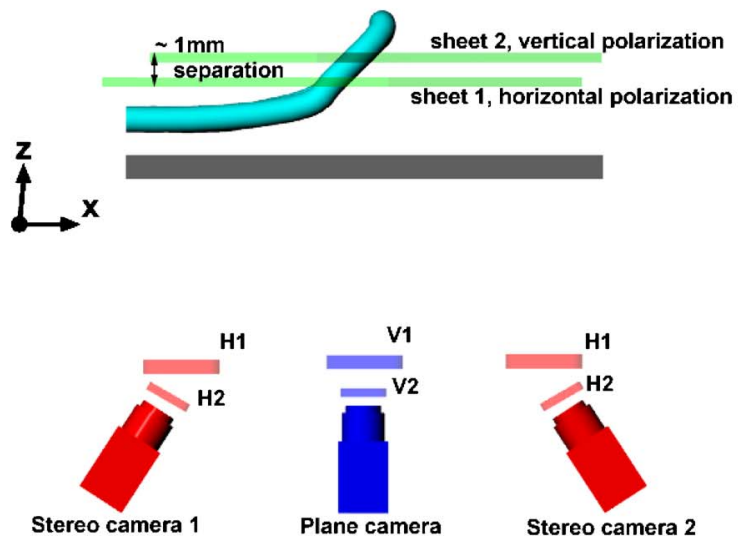

FIG. 1. (Color online) (a) Perspective view and (b) side view of the experimental setup. $\mathrm{H} 1$ and $\mathrm{H} 2$ are linear polarization filters oriented to allow passage of horizontally polarized light. V1 and V2 are linear polarization filters that allow passage of vertically polarized light. The figure was reproduced from Ganapathisubramani et al. (Ref. 16).

three directions are represented as $u, v$, and $w$. All quantities are normalized using $U_{\tau}$ and $\nu$ and denoted with a superscript + .

Two independent PIV systems captured images of light scattered from olive oil droplets (size $\sim 1 \mu \mathrm{m}$ ) simultaneously in neighboring streamwise-spanwise planes separated by $\sim 1.3 \mathrm{~mm}$ (20.5 wall units). As shown in Fig. 1, system 1 is stereoscopic and provides three velocity components over a plane illuminated by sheet 1 , while system 2 uses a single camera to measure the streamwise and spanwise velocity components in a higher plane illuminated by sheet 2. Both sheet pairs were formed from a single SpectraPhysics PIV-400 laser pair. Simultaneous measurements are performed utilizing the polarization property of the laser light sheets to isolate one plane to one camera set. The resolution of the resulting vector fields was nominally $24 \times 24$ wall units, and the total field size was $1.1 \delta \times 1.1 \delta$. A $50 \%$ overlap of the interrogation windows was used while computing the vectors, resulting in a spacing of 12 wall units between adjacent vectors. The single-camera vector fields from the upper plane in liaison with the stereoscopic data from the lower plane were used to compute all velocity gradients in the lower plane. A second order central difference method was used to compute all possible in-plane gradients 
TABLE I. Ensemble averaged flow mean and rms statistics from dual-plane datasets. $\sigma_{\omega_{x}}^{+}, \sigma_{\omega_{y}}^{+}, \sigma_{\omega_{z}}^{+}$are the rms of the fluctuating vorticity components.

\begin{tabular}{lccccccccc}
\hline \hline$z^{+}$ & $z / \delta$ & $\bar{U}^{+}$ & $\sigma_{u}^{+}$ & $\sigma_{v}^{+}$ & $\sigma_{w}^{+}$ & $\overline{u w^{+}}$ & $\sigma_{\omega_{x}}^{+}$ & $\sigma_{\omega_{y}}^{+}$ & $\sigma_{\omega_{z}}^{+}$ \\
\hline 110 & 0.09 & 16.04 & 1.91 & 1.34 & 1.16 & 0.89 & 0.069 & 0.064 & 0.055 \\
575 & 0.53 & 21.7 & 1.41 & 0.97 & 1.12 & 0.44 & 0.054 & 0.057 & 0.032 \\
\hline \hline
\end{tabular}

while a first order forward difference was used to compute the wall-normal gradients of the streamwise and spanwise velocities. Finally, the continuity equation was used to recover the wall-normal gradient of the wall-normal velocity. Details of the experimental set up are given in Ganapathisubramani ${ }^{17}$ and a detailed discussion on the uncertainty and other experimental issues are presented in $\mathrm{Ga}-$ napathisubramani et al. ${ }^{16}$

Datasets comprised of 1200 statistically independent vector fields were acquired in two wall-normal locations, one in the log region at $z^{+}=110$ and the other in the outer wake region at $z^{+}=575(z / \delta=0.53)$. The values of mean and rms statistics of the velocity and vorticity components computed from the dual-plane PIV data at $z^{+}=110$ and $z / \delta=0.53$ are listed in Table I. Figure 2 shows a comparison of the rms values of the three measured vorticity components with the results from various studies available in the literature. These studies include multiprobe hot wire measurements by Balint, Wallace, and Vukoslavcevic ${ }^{18}\left(\operatorname{Re}_{\theta}=2685\right)$, Balint, Wallace, and Vukoslavcevic ${ }^{19} \quad\left(\operatorname{Re}_{\theta}=2080\right)$, Honkan and Andrepoulos ${ }^{11}\left(\operatorname{Re}_{\theta}=2790\right)$, and Lemonis ${ }^{20}\left(\operatorname{Re}_{\theta}=6450\right)$ and a direct numerical simulation (DNS) performed by Spalart ${ }^{21}$ $\left(\operatorname{Re}_{\theta}=1410\right)$. The plots show that the vorticity measurements
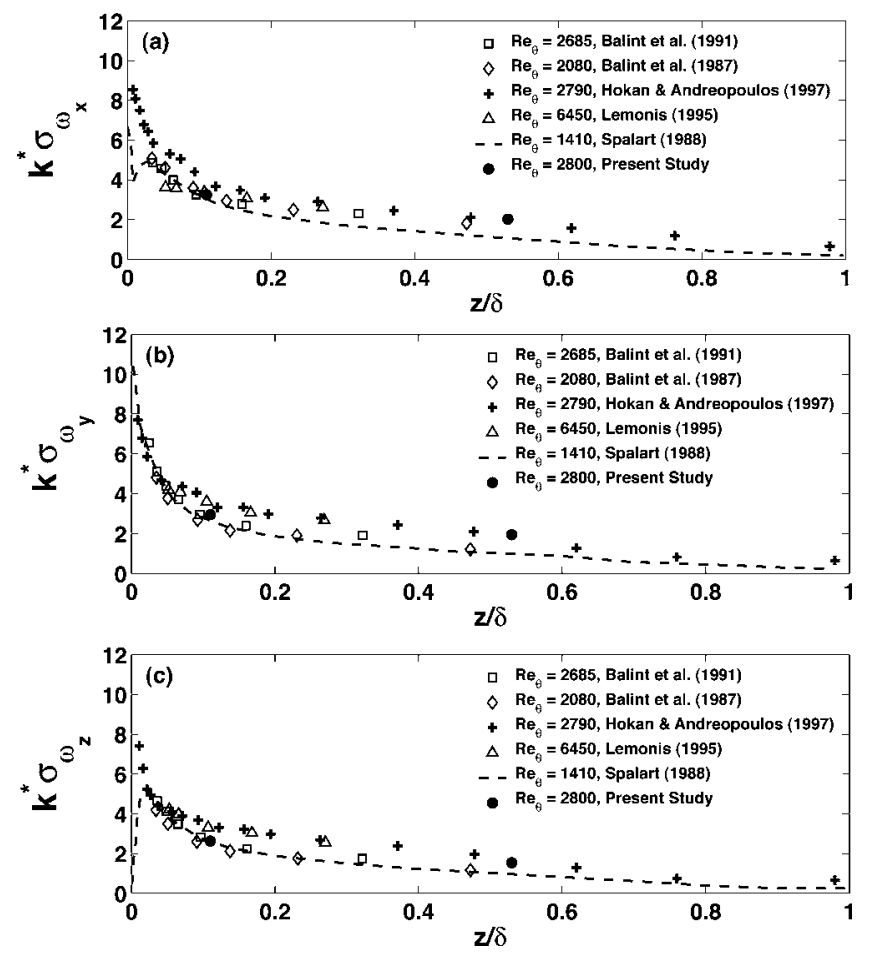

FIG. 2. Comparison of rms values of the three components of vorticity with previous data. (a) $k^{*} \sigma_{\omega_{x}}$, (b) $k^{*} \sigma_{\omega_{y}}$, (c) $k^{*} \sigma_{\omega_{z}}$, where $k^{*}=\delta / U_{\infty}$.
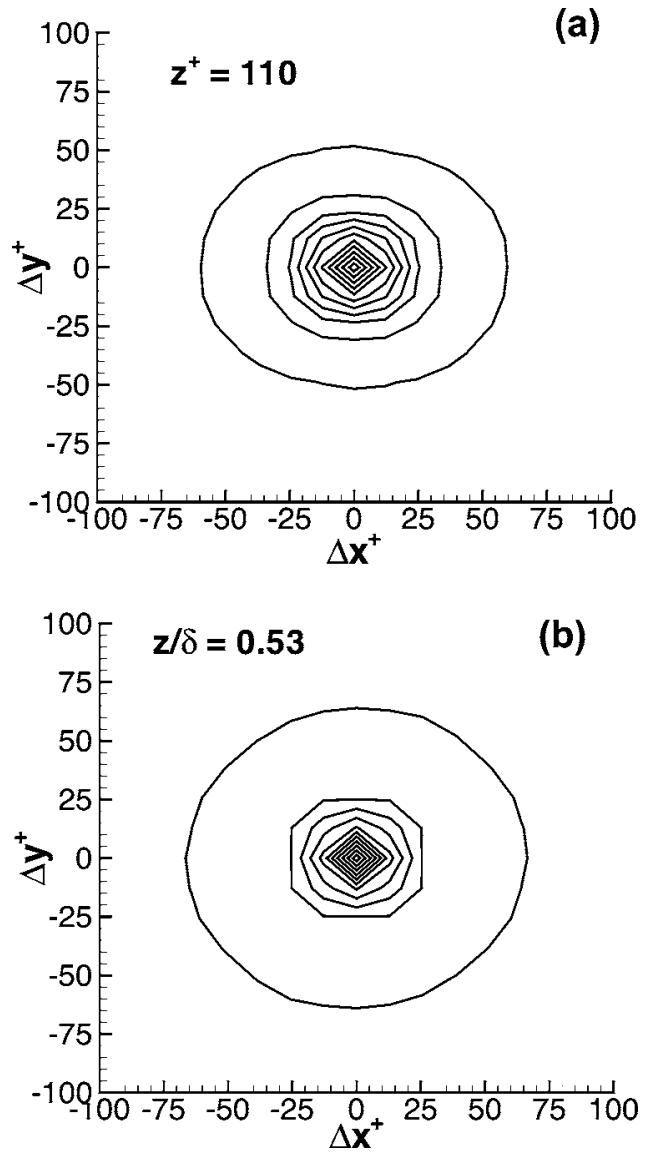

FIG. 3. Two-point auto correlations of $\lambda_{3 \mathrm{D}}$ at $z^{+}=110$ and $z / \delta=0.53$. The contour levels are 0.1 to 1.0 at increments of 0.1 .

from the present study compare very well with previous experimental and DNS data. This observation indicates that the spatial resolution of the study is sufficient to capture the gradients in the flow field.

Further, the validity of the dual-plane-stereo gradients in the log region can be judged by computing the wall-normal gradient of the mean streamwise velocity $(\partial \bar{U} / \partial z)$ and compare this value with the wall-normal gradient predicted by the $\log$ law. For the wall-normal location of $z^{+}=110$, the $\log$ law predicts the gradient to be $90.33 \mathrm{~s}^{-1}$ (for $\kappa=0.41$ ). The average value from an ensemble of 1200 images (with resolution of $100 \times 100$ vectors) is $87.82 \mathrm{~s}^{-1}$. The error in the mean value of the gradient is thus $2.8 \%$ which is well within the expected uncertainty for this first order difference quantity.

\section{CHARACTERISTIC VORTICES: TWO-POINT CORRELATIONS}

The size distribution of vortex structures can be examined by computing two-point autocorrelations of swirl strength $\lambda_{3 \mathrm{D}}$, which is the imaginary part of the eigenvalue of the three-dimensional velocity gradient tensor. Previous studies have shown that this quantity, which isolates regions of fluid swirling about an axis, can be used to visualize vortical structures. ${ }^{12,14}$ Ganapathisubramani et al..$^{22}$ present a complete description of the technique followed in computing the 
correlations. Figure 3 shows the autocorrelation of $\lambda_{3 \mathrm{D}}^{+}$at $z^{+}=110$ and $z / \delta=0.53$. The extent of the outermost contour levels can be interpreted as a representative length scale of the largest vortex cores. This figure does not show any discernible difference in the location of higher contour levels in the $\log$ and the outer region. However, the extent of the lowest contour level indicates that the largest structures are larger in the outer region than in the log region. Also, the shapes of the lower contour levels indicate that larger structures are more elongated along the streamwise direction in the log region than in the outer wake region. This trend is consistent with the presence of eddies whose inclination angle increases with increasing wall-normal distance. Note however that these correlation plots include contributions from all possible vortex cores with various inclinations and orientations. Hence, arriving at a possible conclusion on the orientation of individual eddy cores is not possible.

The shape of the cores can be studied further by separating $\lambda_{3 \mathrm{D}}$ correlations into four separate groups based on the four quadrants in the $\left(\omega_{x}, \omega_{z}\right)$ plane to distinguish between cores that are leaning forward and cores that are leaning backward (against the freestream direction):

$$
\begin{aligned}
\lambda_{1} & =\lambda_{3 \mathrm{D}} \text { for } \omega_{x}>0, \omega_{z}>0 \\
& =0 \text { otherwise, } \\
\lambda_{2} & =\lambda_{3 \mathrm{D}} \text { for } \omega_{x}<0, \omega_{z}>0 \\
& =0 \text { otherwise, } \\
\lambda_{3} & =\lambda_{3 \mathrm{D}} \text { for } \omega_{x}<0, \omega_{z}<0 \\
& =0 \text { otherwise, } \\
\lambda_{4} & =\lambda_{3 \mathrm{D}} \text { for } \omega_{x}>0, \omega_{z}<0 \\
& =0 \text { otherwise. }
\end{aligned}
$$

Two-point autocorrelations for each of these separated swirl strengths were computed with the goal of isolating a dominant vortex core shape for various streamwise-wall-normal orientations in the streamwise-spanwise plane.

The autocorrelation contours for these separated swirl parameters at both wall-normal locations are shown in Fig. 4. The $\lambda_{1}$ autocorrelations at both wall-normal locations [Figs. 4(a) and 4(b)] are elongated in the streamwise direction, and the major axes of the elliptical contour shapes are angled away from the $x$ axis. Similarly, the $\lambda_{3}$ autocorrelations also show elongated contours with an inclined major axis. The autocorrelations of the forward-leaning groups $\left(\lambda_{1}\right.$ and $\left.\lambda_{3}\right)$ when taken together, seem to imply that vortex cores are angled inwards towards each other. This feature is also found in the autocorrelations of the backward-leaning groups as seen in Figs. 4(c) and 4(d). The above noted feature of the autocorrelations does not imply the absence of individual forward-leaning or backward-leaning structures of other spanwise orientations. Each correlation simply isolates a representative shape of the structure. Another interesting note inferred from these correlation plots is that the backward-
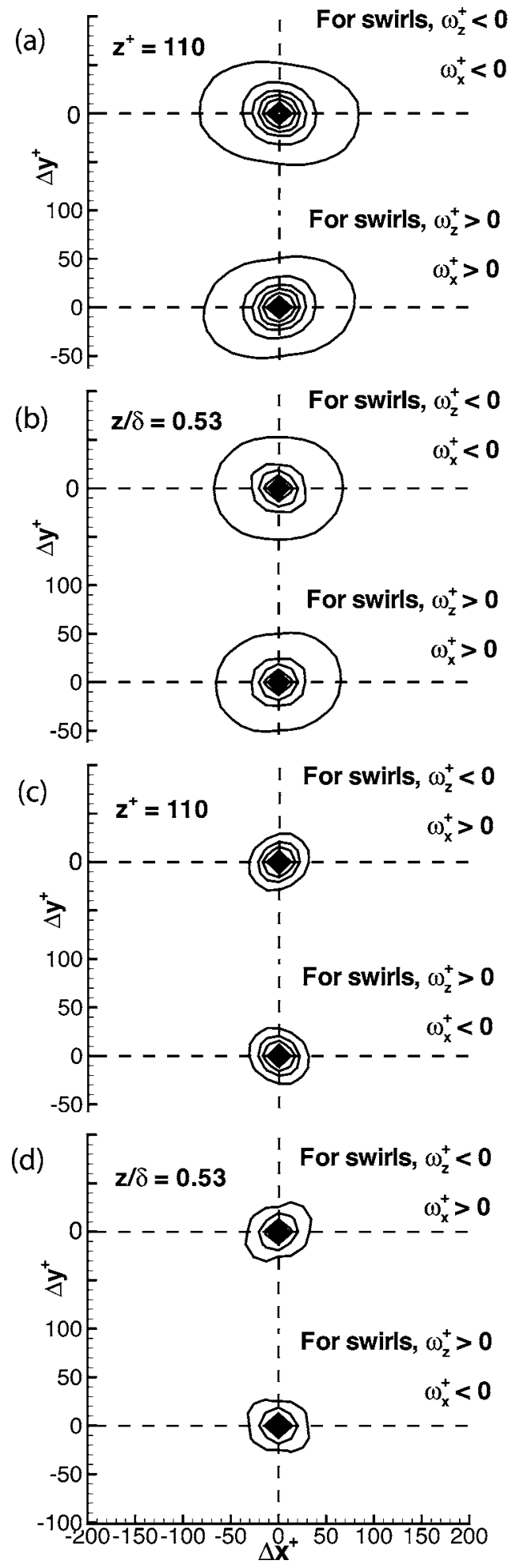

FIG. 4. Two-point autocorrelations of $\lambda_{3 \mathrm{D}}$ separated into four quadrants according to $\omega_{x}$ and $\omega_{z}$. $[(\mathrm{a})$ and (c) $] z^{+}=110 ;[(\mathrm{b})$ and (d) $] z / \delta=0.53$. The contour increments are 0.1 and the outermost contour is equal to the increment.

leaning cores are typically small in core size, while the forward-leaning cores extend through a range of sizes, small to large.

A hypothetical model for the geometric structure of a representative vortex core can be constructed based on the shapes of the contours in Fig. 4. Figure 5(a) shows a sche- 


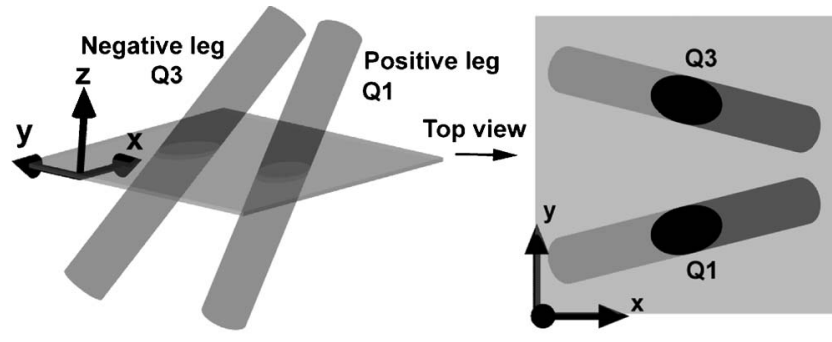

(a)

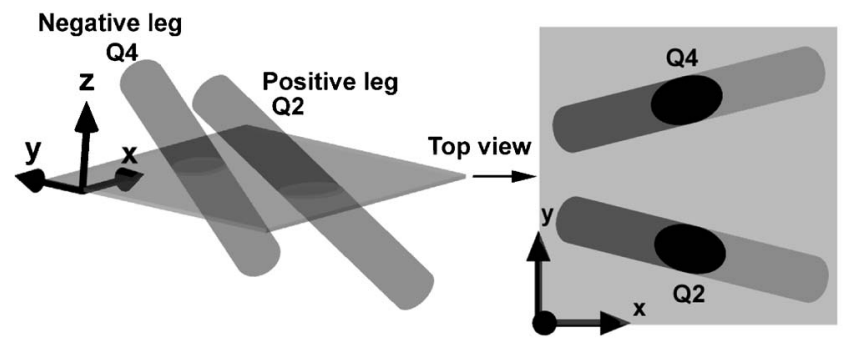

(b)

FIG. 5. Hypothetical model of an average vortex in a turbulent boundary layer constructed based on two-point correlations of swirl strength.

matic representation of a forward-leaning " $\Lambda$ " shaped eddy and its projection on the streamwise-spanwise plane. The projections in the first and third quadrants of the forwardleaning eddy are qualitatively similar to the contour shapes in Figs. 4(a) and 4(b). Figure 5(b) illustrates a backwardleaning " $\Lambda$ " shaped structure and its projection onto the $x-y$ plane. The projections of the eddy are similar to the contour shapes in Figs. 4(c) and 4(d).

\section{VORTEX GEOMETRY: STATISTICAL AND INSTANTANEOUS RESULTS}

The dual-plane data can be used to compute the inclination angle of any individual vortex structure intersecting the measurement plane by determining the orientation of the vorticity vector averaged over the region of the vortex core. It is important to distinguish this concept from an instantaneous vorticity vector angle at a point. ${ }^{11}$ The instantaneous vorticity field contains many small-scale fluctuations. By computing the vorticity vector averaged over a region identified as a vortex core by the swirl strength $\lambda_{3 \mathrm{D}}$, the small-scale variations are averaged out, leading to determination of the orientation of the vortex core. This orientation can then be interpreted as the local inclination of that vortex. A vortex core region is isolated using a region growing algorithm that locates connected regions of swirl greater than a specified threshold. The technique and details of identifying a vortex core to compute the inclination angle are described in the following section.

\section{A. Vortex identification technique}

Vortex inclination angles were found by computing the average vorticity vector in a connected region of swirl. The connected region was found using a region growing algorithm that searched for connected points of swirl strength greater than a certain threshold. The algorithm works with any scalar vortex identification parameter. Previous studies have compared and contrasted various vortex identification parameters, ${ }^{13,23}$ but there is no general consensus in the literature on an optimal parameter to isolate vortex cores. In this study, $\lambda_{3 \mathrm{D}}$ and $\lambda_{2 \mathrm{D}}$ were used to identify vortex cores (where $\lambda_{2 \mathrm{D}}$ is the two-dimensional swirl strength computed using a reduced tensor that contains the in-plane velocity gradients only $\left.{ }^{24}\right)$. The two-dimensional swirl strength $\left(\lambda_{2 \mathrm{D}}\right)$ isolates regions that are swirling about an axis with a component aligned normal to the plane of measurements. If the inclination of vortices with respect to the wall is small, $\lambda_{2 \mathrm{D}}$ does a poor job at identifying them.

The details of the algorithm to identify the vortex cores are described below.

- Step 1: All points of local maxima in the swirl strength $\left(\lambda_{3 D}\right.$ or $\left.\lambda_{2 D}\right)$ field are identified and marked.

- Step 2: The points of local maxima greater than a given threshold are isolated.

The points identified as local maxima also include points that are part of weak vortex structures and measurement noise. In order to filter out the weak structures, a threshold based on the maximum value of swirl strength in the dataset of 1200 instantaneous fields is utilized. This threshold was fixed at $10 \%$ of the maximum value of scalar parameter in the dataset. Various tests were performed to arrive at an acceptable threshold value for swirl strength. Zhou et al. ${ }^{12}$ used various percentages of a maximum value to visualize vortex structures in their DNS datasets. The authors concluded that over a certain range of threshold values, the general shape of a vortex did not change; however, increasing the threshold beyond a certain limit decreased the influence of background noise. Details on the effect of threshold value on the results are discussed in Sec. IV C.

- Step 3: The points of local maxima are used as seed points for a region growing algorithm that isolates a connected region of swirl with values greater than the specified threshold.

Figure 6 illustrates the working of the region growing algorithm. Figure 6(a) shows a plot of $\lambda_{3 \mathrm{D}}^{+}$at $z^{+}=110$. The maximum value of $\lambda_{3 \mathrm{D}}^{+}$at this wall-normal location was 0.35 , and the threshold chosen was 0.035 (10\% of the maximum). Figure 6(b) shows the connected regions identified by the region growing algorithm. This clearly shows that the algorithm captures all the structures with swirl values greater than the threshold. It must be noted that the algorithm cannot distinguish between (or separate) cores that are in close proximity or overlapping. This issue, which could affect the distribution of the angles computed, is discussed in detail in Sec. IV C.

- Step 4: An identified connected region must include a minimum area to be accepted as a vortex core. If the number of points in the region is less than a threshold value, then the connected region is not included in the angle computation described in step 5. 

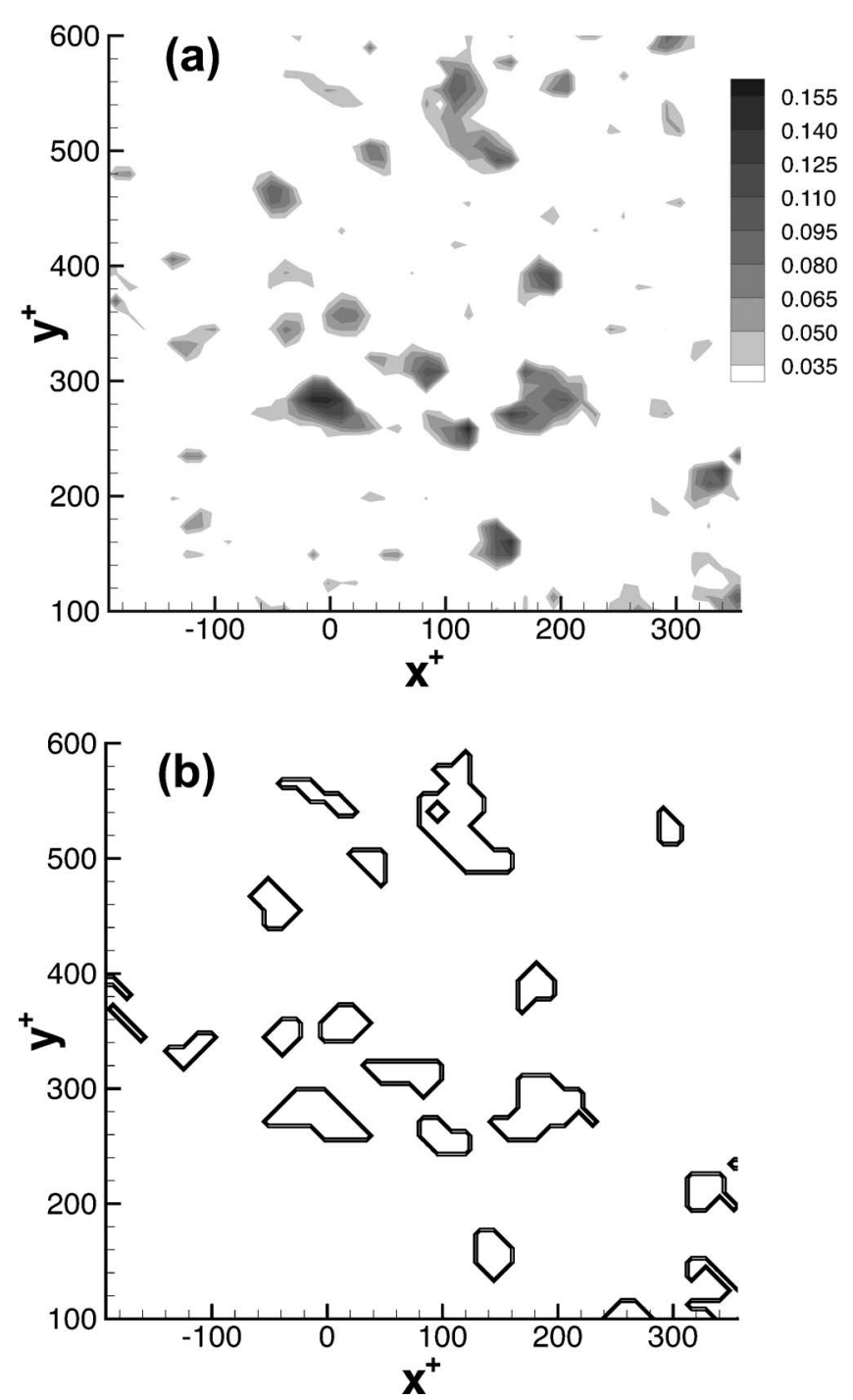

FIG. 6. Performance of region growing algorithm. (a) $\lambda_{3 \mathrm{D}}$; (b) identified regions.

A minimum number of points for the identification of a vortex core were necessary to filter any contribution from measurement uncertainty and other noise since neither the PIV data nor the scalar parameter used to identify vortex cores were subject to any smoothing or filtering. In this study the threshold value was fixed at five contiguous points (this translates to an area of approximately 1000 square wall units). The specified threshold also results in good convergence of the local core vorticity vector from which the angles are computed. In Fig. 6(b), it can be observed that the threshold filters small areas of strong $\lambda_{3 \mathrm{D}}$. The effect of area threshold is also discussed in detail in Sec. IV C.

- Step 5a: Average values of the three components of vorticity are computed in an accepted connected region. (These values are then used to compute the inclination angles made by that specific vortex core with various planes and axes.)

- Step 5b: The average value of at least one component of vorticity must be greater than the standard deviation of that component (i.e., strong value) for the angles to be used in the distributions.

The threshold on swirl strength also ensures the exclusion of areas with weak enstrophy values (where enstrophy is the magnitude of vorticity, $\sqrt{\omega_{x}^{2}+\omega_{y}^{2}+\omega_{z}^{2}}$ ). However, three relatively weak vorticity components might result in enstrophy/swirl values that exceed the threshold and therefore would be identified as vortex cores by the algorithm. Therefore, the above mentioned step was added to prevent vortex cores with three individually weak vorticity components from contributing to the probability distributions of orientations. This step ensures the presence of at least one strong component of vorticity in all of our angle computations.

- Step 5c: A given projection angle (i.e., $\theta_{y x}, \theta_{y z}$, and $\theta_{x z}$ ) is computed only if the average vorticity value of at least one component used in computing the angle is larger than its standard deviation (i.e., $\bar{\omega}>1.0 \sigma_{\omega}$ ).

This additional condition was imposed in order to avoid contributions to the probability density function (pdf) of specific projection angles from weak vorticity values. For example, a spanwise oriented vortex core would have $\omega_{x}$ and $\omega_{z}$ values nearly equal to zero, however, such a core would reveal a projection angle of close to $45^{\circ}$ in the $x-z$ plane (as $\omega_{x} \approx \omega_{z}$ ). This is markedly different from a forward-leaning vortex core that is inclined at $45^{\circ}$ to the $x-z$ plane.

- Step 6: Population densities and probability distributions of the various inclination angles are computed. The uncertainty in angles computed from weak vorticity values is much larger than the uncertainty in angles computed with stronger vorticity components. Therefore, step 5 in the vortex identification algorithm was introduced as a precautionary measure to prevent relatively weak values of vorticity from contributing to the distributions. The presence of step 5 in the algorithm decreased the number of the vortex cores included in the distributions, however it did not alter the shapes of the distributions significantly.

\section{B. Vorticity covariances}

Ong and Wallace ${ }^{10}$ performed a joint probability density analysis of various components of vorticity obtained using hot-wire measurements to study the dominant vortex orientation. This analysis was similar to the quadrant splitting analysis of Reynolds shear stress developed independently by Wallace, Eckelmann, and Brodkey ${ }^{25}$ and Willmarth and $\mathrm{Lu}^{26}$

This analysis involved determining the joint probability density function (JPDF), $P(a, b)$ of any two variables $a$ and $b$, where

$$
\overline{a b}=\iint_{-\infty}^{\infty} a b P(a, b) d a d b .
$$

This integral of the covariance integrand, $a b P(a, b)$ over a differential area, represents the contribution of that particular simultaneous combination of sign and magnitude of $a$ and $b$ to the covariance $\overline{a b}$. Wallace and Brodkey ${ }^{27}$ plotted con- 

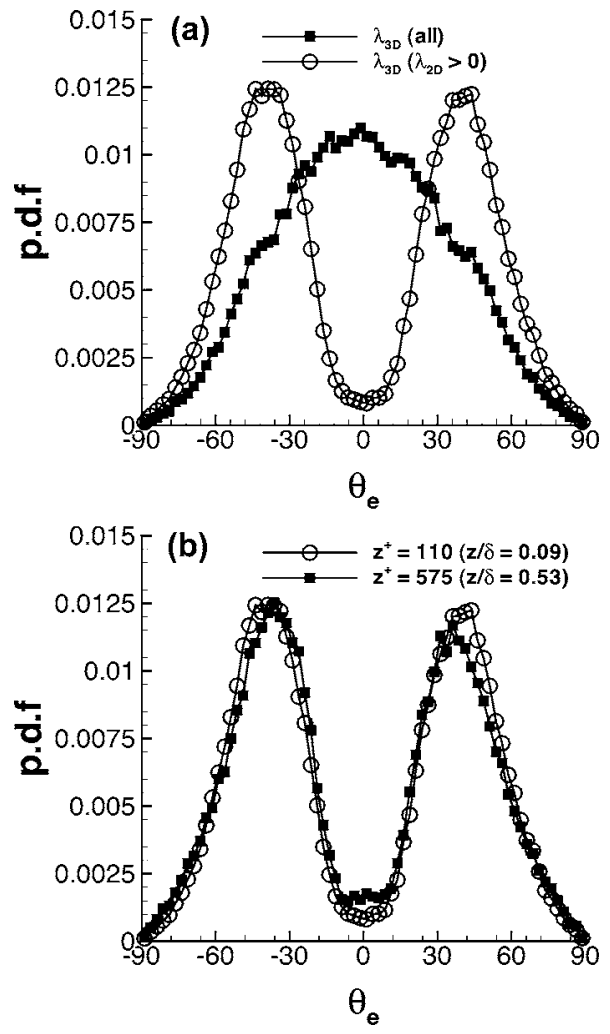

FIG. 7. Pdf of elevation angle $\left(\theta_{e}\right)$ at $z^{+}=110$ and (b) $\theta_{e}$ at $z^{+}=110$ and $z / \delta=0.53$.

tours of $P(u, w)$ and $u w P(u, w)$ (covariance) to study the dominant contributors to the Reynolds shear stress.

Ong and Wallace ${ }^{10}$ used a similar analysis. However they used joint probability density functions and covariances of various vorticity components to study the structure of a boundary layer. The authors used the covariances of $\left(\omega_{x}, \omega_{y}\right)$, $\left(\omega_{x}, \omega_{z}\right)$, and $\left(\omega_{y}, \omega_{z}\right)$ to determine a dominant structure. An identical analysis was performed in this study and its results are compared to the distributions of the projection angles obtained from analyzing the instantaneous vortex cores. The results are described in greater detail in the following section.

\section{Vortex angles}

Figure 7(a) shows the probability density function (pdf) of the angle $\theta_{e}$ made by the vortex cores at $z^{+}=110\left(\theta_{e}\right.$ is the angle made by the vorticity vector with the $x-y$ plane, $-90^{\circ}<\theta_{e}<90^{\circ}$. This angle is also called the elevation angle). This distribution (shown as square symbols) includes a wide range of structure angles at this wall-normal location. Note that many structures have small inclination angles. Further study, including the investigation of the azimuthal angle made by the projection of the vorticity vector onto the $x-y$ plane with the $x$ axis, reveals that most $\lambda_{3 \mathrm{D}}^{+}$regions with small inclinations are spanwise structures indicative of heads of smaller hairpin vortices or other in-plane oriented vortices. In order to obtain the inclination angles of cores that are not spanwise heads or streamwise legs, the average vorticity vector in isolated regions of $\lambda_{3 \mathrm{D}}^{+}$that include $\lambda_{2 \mathrm{D}}^{+}$(i.e., $\lambda_{2 \mathrm{D}}^{+}$ $>0$ ) was computed.
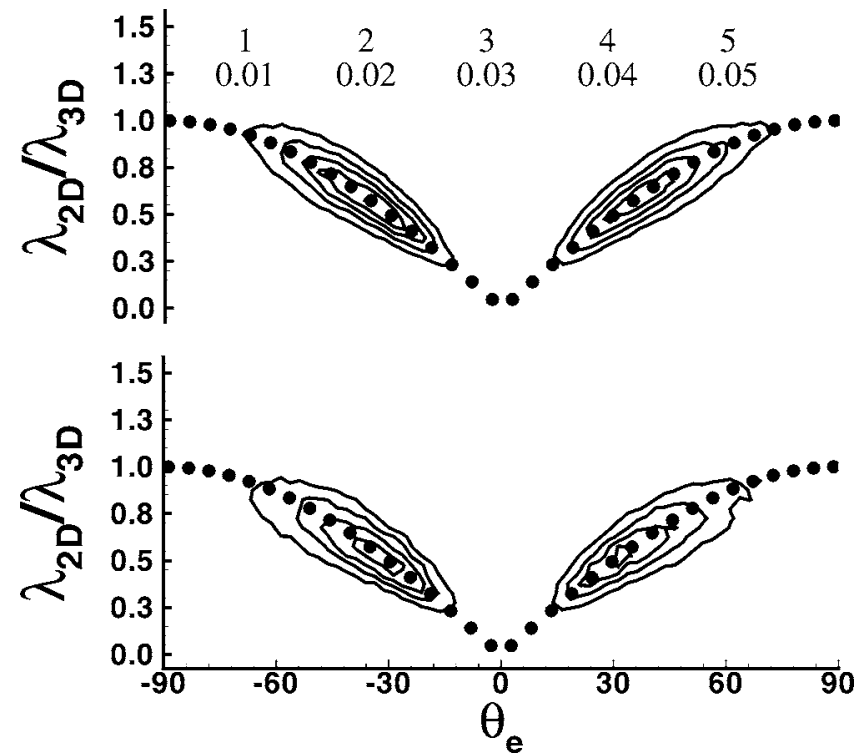

FIG. 8. Joint pdf of inclination angle and $\lambda_{2 \mathrm{D}} / \lambda_{3 \mathrm{D}}$ at $z^{+}=110$ (top) and $z / \delta=0.53$ (bottom), the dotted line is the function $\lambda_{2 \mathrm{D}} / \lambda_{3 \mathrm{D}}=\left|\sin \theta_{e}\right|$.

Since $\lambda_{2 \mathrm{D}}^{+}$is computed using only the in-plane velocity gradients, it only identifies regions that are swirling about an axis normal to the measurement plane. Therefore this additional criterion filters out spanwise and streamwise structures (since they do not contribute to $\lambda_{2 \mathrm{D}}^{+}$), and enables the investigation of the wall-normal orientation of the remaining vortex cores. The resulting pdf, shown by circles in Fig. 7(a), which no longer has a peak at zero inclination angle, characterizes the elevation angle of vortices that are not spanwise heads, streamwise legs or vortices with any other in-plane $(x-y$ plane) orientation. In all further pdf plots of vortex angles, spanwise heads and streamwise legs are not included in the pdf (i.e., $\lambda_{2 \mathrm{D}}^{+}>0$ is required).

Figure 7(b) shows the comparison of the inclination angles $\left(\theta_{e}\right)$ at $z^{+}=110$ and $z / \delta=0.53$. This pdf yields peaks at $\pm 38^{\circ}$ for $z^{+}=110$ and $\pm 33^{\circ}$ for $z / \delta=0.53$. This result suggests that the dominant inclination angle decreases slightly with wall-normal distance. Note, however, that the peaks are broad, and a wide range of inclination angles is present at each location.

Figure 8 is a plot of the joint probability distribution of the ratio of $2 \mathrm{D}$ swirl strength to $3 \mathrm{D}$ swirl strength and the inclination angle $\left(\theta_{e}\right)$. It is worth noting that, mathematically, $\lambda_{2 \mathrm{D}}$ will always be less than or equal to $\lambda_{3 \mathrm{D}}$ for any orientation. The distribution indicates a unique relationship between this ratio and the inclination angle of vortices with respect to the cutting plane ( $x-y$ plane in this instance). Velocity fields induced around idealized hairpin vortices (with and without curvature) were computed using Biot-Savart calculations to calculate the ratio $\lambda_{2 \mathrm{D}} / \lambda_{3 \mathrm{D}}$ as a function of the vortex rod angle. The results from this computation suggest that the ratio of the two swirl strengths varies as $\left|\sin \theta_{e}\right|$. The value of this ratio from the experiments follows this theoretical finding as seen in Fig. 8. Note that this plot does not reveal any information about the streamwise or spanwise oriented structures, since the ratio was computed only for cores where $\lambda_{2 \mathrm{D}}$ was greater than zero. The fact that the distribution is dense 


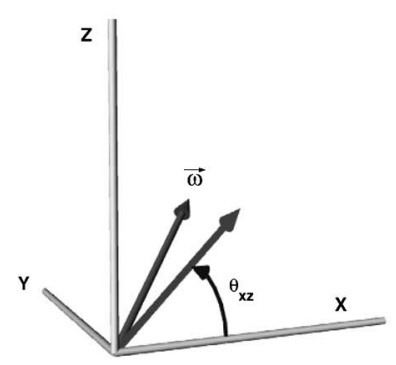

(a)

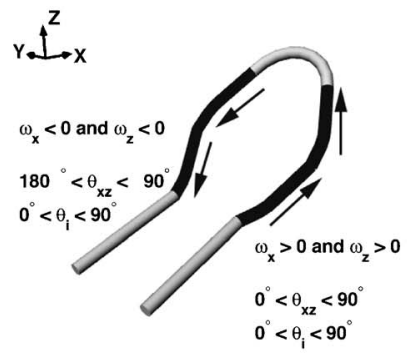

(c)

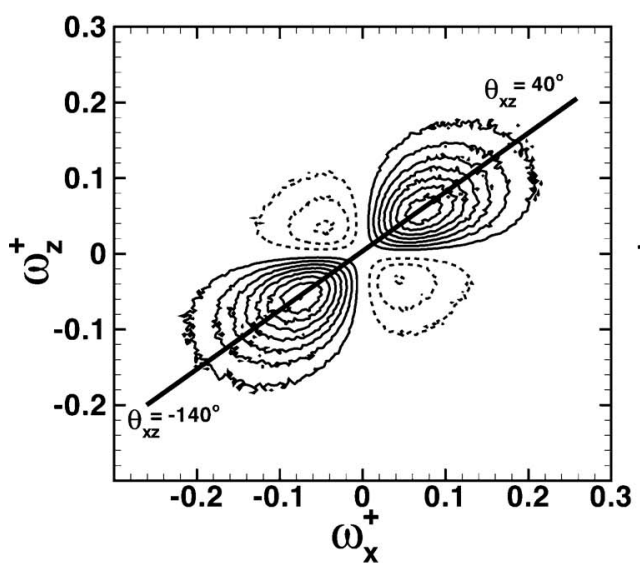

(e)

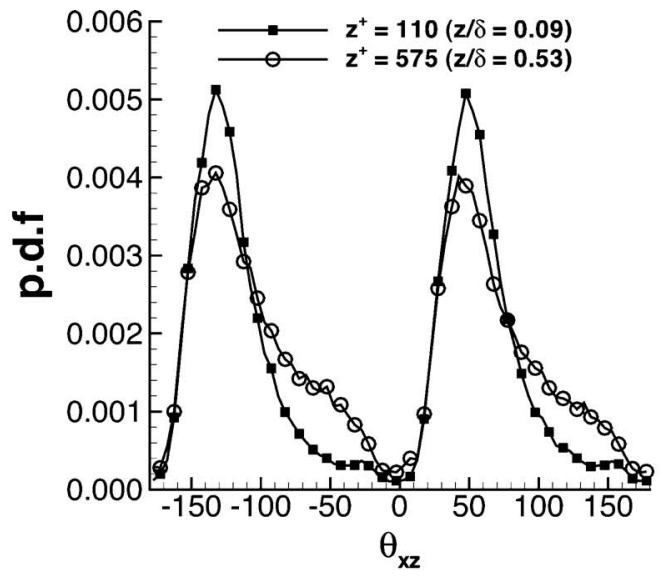

(b)

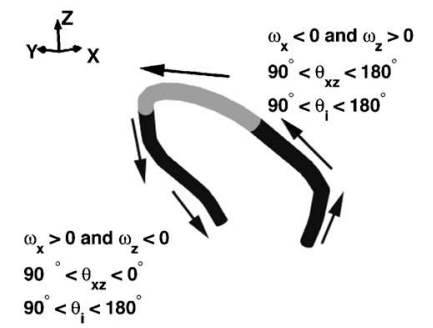

(d)

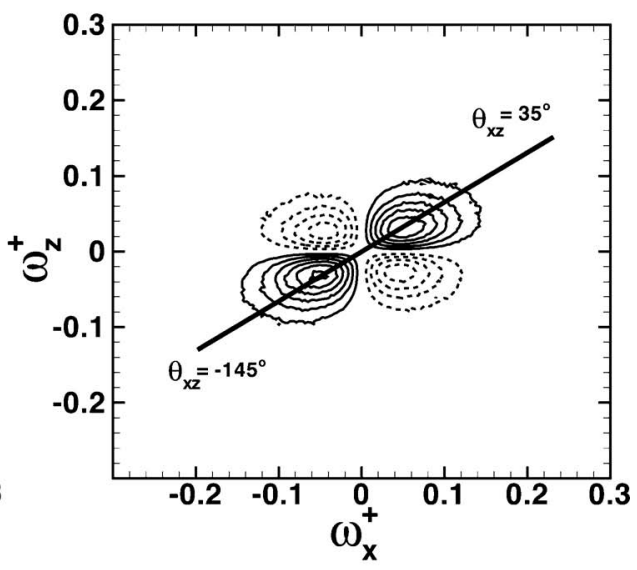

(f)
FIG. 9. (a) Schematic representation of $\theta_{x z}$, (b) pdf of $\theta_{x z}$, (c) and (d) schematic representations of a hairpin loop. Covariance of $\left(\omega_{x}, \omega_{z}\right)$ at (e) $z^{+}$ $=110$ and (f) $z / \delta=0.53$. The contour increment is \pm 0.01 and the first level shown is \pm 0.01 . Negative contours are shown with dotted lines and the zero contour is not shown. in the angle range $20^{\circ}<\theta_{e}<50^{\circ}$ at both wall-normal locations indicates that most vortex cores are inclined in that range of angles.

The projections of the vorticity vector in the $x-z, x-y$, and $y-z$ planes can be used to compute the projection angles in respective planes. A schematic definition of the $\theta_{x z}$, the angle made by the projection of the vorticity vector in the $x-z$ plane with the positive $x$ axis is shown in Fig. 9(a). The probability distribution of $\theta_{x z}$ is shown in Fig. 9(b). Figure 9(b) reveals peaks at $\theta_{x z} \approx 45^{\circ}$ and $-135^{\circ}$, respectively at both wall-normal locations. This is analogous to the forwardleaning positive and negative legs of a hairpin-type vortex as shown in Fig. 9(c). Note that the symmetric hairpin sketches in Fig. 9(c) and other subsequent figures represent a simplified "average" structure used to aid the discussion. We do not mean to imply that all structures are symmetric or that all structures can be represented by simple hairpins. It is clear from the distribution of $\theta_{x z}$ that the vortex cores identified possess a wide range of inclination angles. Backwardleaning cores as shown in Fig. 9(d) contribute to values of $\theta_{x z}$ in the range $90^{\circ}<\theta_{x z}<180^{\circ}$ and $-90^{\circ}<\theta_{x z}<0^{\circ}$. However, the peaks at $45^{\circ}$ and $-135^{\circ}$ indicate that the logarithmic and outer regions of the boundary layer are dominated by forward-leaning vortex cores. This result is consistent with the angle computed from the joint pdf analysis of $\left(\omega_{x}, \omega_{z}\right)$. Figures 9(e) and 9(f) show the covariance of $\left(\omega_{x}, \omega_{z}\right)$ at $z^{+}$ $=110$ and $z / \delta=0.53$. The covariance plots reveal the dominance of the contributions from quadrant $1\left(\omega_{x}>0, \omega_{y}>0\right)$ and quadrant $3\left(\omega_{x}<0, \omega_{z}<0\right)$ consistent with the distribution of $\theta_{x z}$, shown in Fig. 9(b). The angles of inclination made with the positive $x$ axis are inferred from the peaks in 

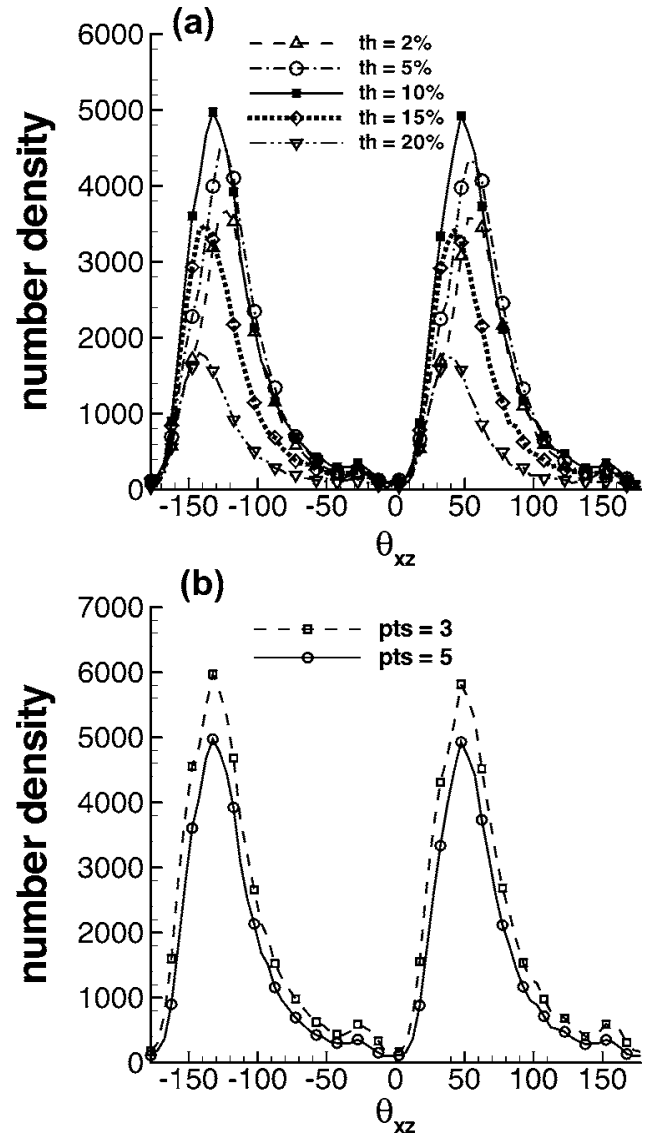

FIG. 10. Effect of threshold on swirl strength value and the area occupied. The figures show number densities of $\theta_{x z}$ for various thresholds of (a) swirl strength and (b) core area.

covariances. The angles at $z^{+}=110$ were computed as $39^{\circ}$ and $-143^{\circ}$ and the angles at $z / \delta=0.53$ are $31^{\circ}$ and $-150^{\circ}$. This is consistent with the findings of Ong and Wallace ${ }^{10}$ and with the presence of forward leaning vortex filaments.

Figure 10(a) shows the effect of swirl strength threshold (used in step 2 of the vortex core identification algorithm) on the number density of the projection angle $\theta_{x z}$ at $z^{+}=110$. The figure indicates that the number of cores identified initially increases and then decreases with increasing threshold. For small values of the threshold, multiple adjacent cores are identified as one, reducing the total number of cores identified. Increasing the threshold isolates the adjacent cores, and the number of cores identified increases up to a certain value of the threshold (found to be $10 \%$ of maximum value). Beyond this value the number density starts decreasing since the number of cores with swirl strength values greater than the threshold is smaller. Figure 10(a) also illustrates that the most probable and mean angles decrease with increasing threshold suggesting that stronger cores have a relatively lower inclination angle. Higher thresholds also reduce the number density of backward-leaning cores $\left(90^{\circ}<\theta_{x z}<180^{\circ}\right.$ and $-90^{\circ}<\theta_{x z}<0^{\circ}$ ) indicating that backward-leaning cores possess relatively lower strength. A balance must be struck between the threshold used, the number of cores identified and the peak angle identified from the distribution. In all of the results, a threshold of $10 \%$ of the maximum value of swirl strength was used to identify the vortex cores since this
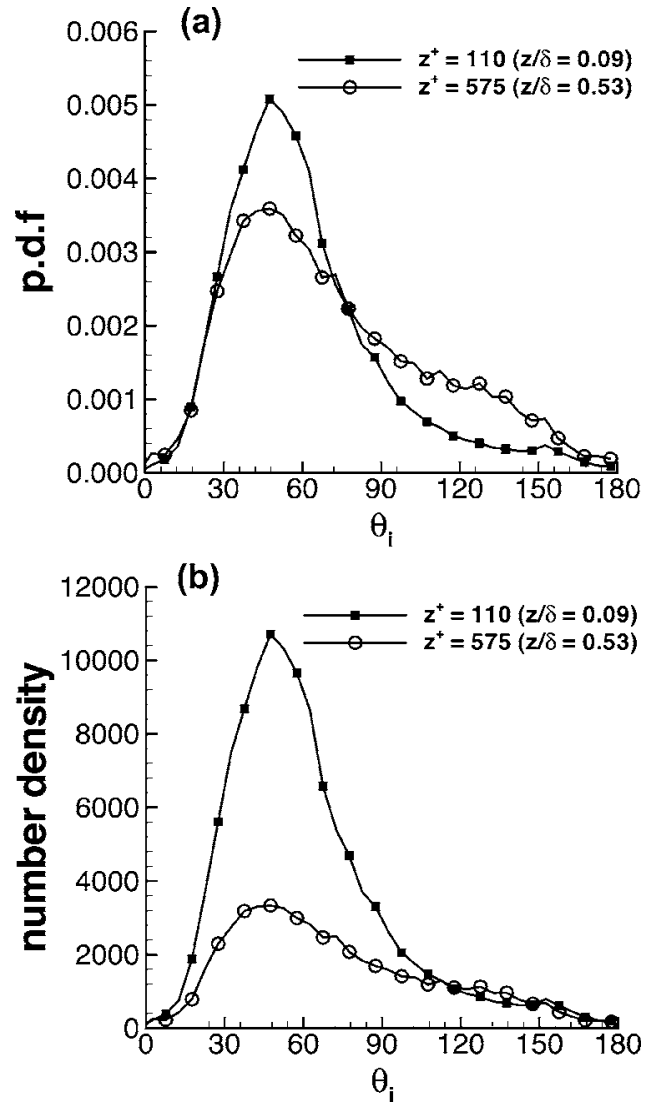

FIG. 11. Eddy inclination angle $\left(\theta_{i}\right)$; (a) pdf and (b) absolute number density.

value of the threshold isolates the maximum number of cores with minimal agglomeration of adjacent cores. Figure 10(b) shows the effect of the area threshold on the probability density distribution of $\theta_{x z}$ at $z^{+}=110$. The number of points used does not seem to affect the peak values of the pdf or the shape of the distribution. The key difference is that a smaller area threshold (three connected points $\sim 600$ square wall units) identifies a larger number of cores.

The angles in the first and third quadrants (forwardleaning) and the angles in the second and fourth quadrants (backward-leaning) can be combined to represent a single eddy inclination angle $\left(\theta_{i}\right)$ that varies from $0^{\circ} \leqslant \theta_{i} \leqslant 180^{\circ}$. This eddy inclination angle is computed on the assumption that eddies are symmetric about an $x-z$ plane. All forwardleaning cores (both positive and negative legs as shown in Fig. 5) are accumulated into one group while all backwardleaning cores are grouped together. The resulting pdf of the eddy inclination angle is given in Fig. 11(a), and it has peaks at angles of $46^{\circ}$ at $z^{+}=110$ and $42^{\circ}$ at $z / \delta=0.53$. These results are comparable to an average $45^{\circ}$ hairpin inclination as suggested by various researchers in the past. ${ }^{5}$ Figure 11(b) shows the absolute number density of $\theta_{i}$ at the two wallnormal locations. Clearly, a larger total number of vortex cores were found in the log region than in the wake region. The ratio of the area under the curve for $0^{\circ}<\theta_{i} \leqslant 90^{\circ}$ and $90^{\circ}<\theta_{i} \leqslant 180^{\circ}$ was computed to study the relative density of forward and backward-leaning cores. This ratio was found to be 5.3 at $z^{+}=110$ and 2.4 at $z / \delta=0.53$. Thus the relative 


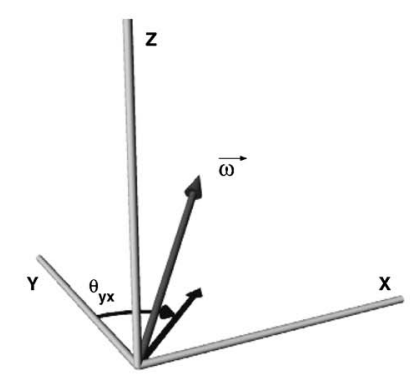

(a)

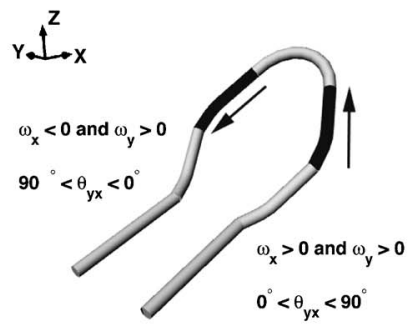

(c)

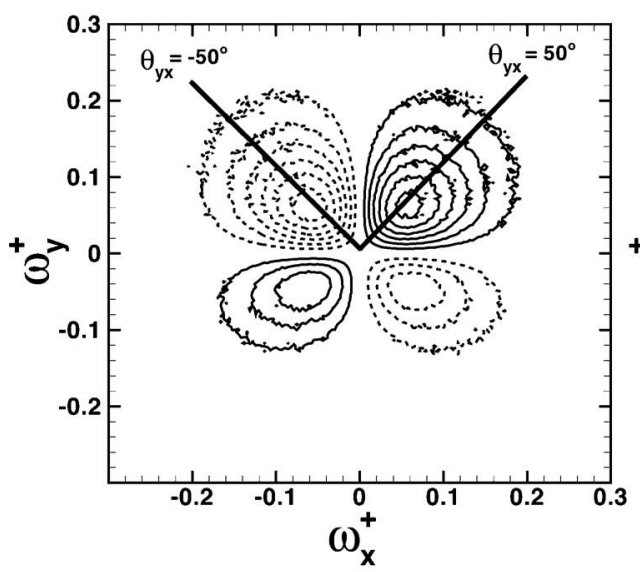

(e)

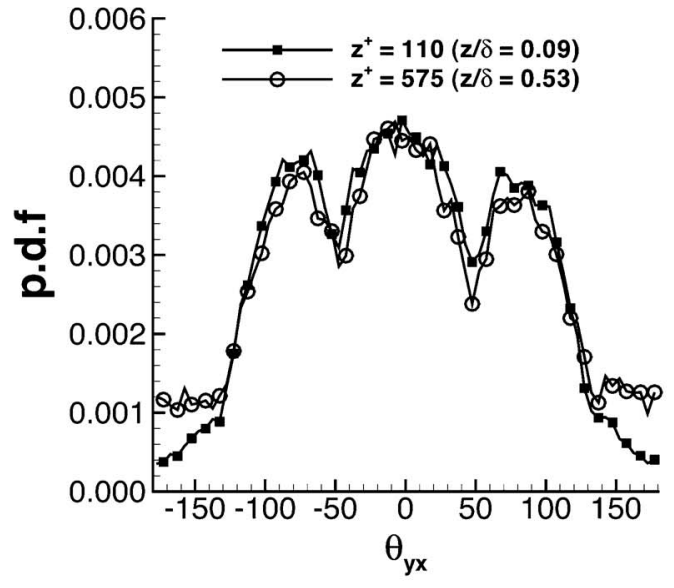

(b)

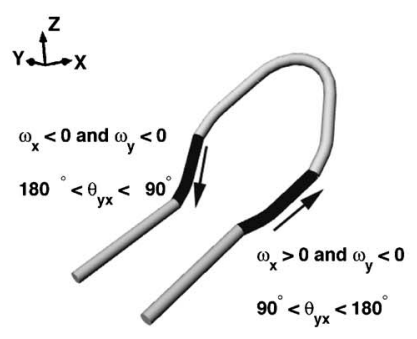

(d)

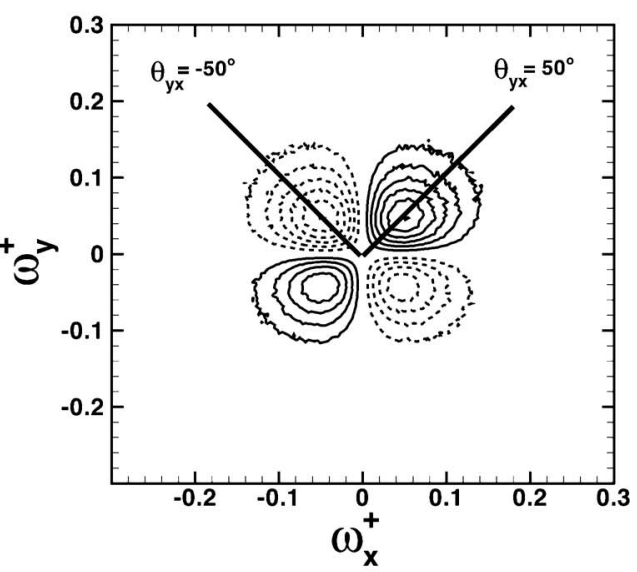

(f)
FIG. 12. (a) Schematic representation of $\theta_{y x}$, (b) pdf of $\theta_{y x}$, (c) and (d) schematic representations of a hairpin loop. Covariance of $\left(\omega_{x}, \omega_{y}\right)$ at (e) $z^{+}$ $=110$ and (f) $z / \delta=0.53$. The contour increment is \pm 0.01 and the first level shown is \pm 0.01 . Negative contours are shown with dotted lines and the zero contour is not shown. number of forward-leaning cores is much larger in the log region than in the wake region which is congruent with the results from the covariance plots in Figs. 9(e) and 9(f) and consistent with the findings of Ong and Wallace. ${ }^{10}$ An interesting point to also note from Fig. 11(b) is that the number density of the backward-leaning cores in the log region and the wake region remains relatively constant thereby suggesting universality in the number of backward-leaning cores.

Having established that the boundary layer is comprised of predominantly forward-leaning eddies, the geometric structure of these eddies can be studied further by examining the projection angles in the $x-y$ and $y-z$ planes. Figure 12(a) shows the definition of the projection angle $\theta_{y x}$, which is the angle made by the projection of the vorticity vector in the $x-y$ plane with the positive $y$ axis. Figure 12(b) shows the pdf of $\theta_{y x}$ at both wall-normal locations. The figure clearly reveals that a large percentage of structures have orientations in the range $-110^{\circ}<\theta_{y x}<110^{\circ}$. The distributions at both wall-normal locations have three peaks. The first peak is at $\theta_{y x} \approx 0^{\circ}$, presumably caused by heads of hairpin loops. Hairpin loops with inclination angles $\left(\theta_{i}\right)$ of nearly $\pm 90^{\circ}$ would also contribute to the $\theta_{y x}$ peak at $0^{\circ}$. The second and third peaks at $\theta_{y x} \approx 75^{\circ}$ and $-75^{\circ}$ could be caused by the positive and negative " $\Lambda$ " shaped necks (or vortex rods that are tilted inwards) as shown in Fig. 12(c). Honkan and Andreopoulos ${ }^{11}$ computed $\theta_{y x}$ from instantaneous vorticity vectors based on multiprobe hot wire measurements and found peaks at approximately $60^{\circ}$ and $-60^{\circ}$ at $z^{+}=13$. This finding, in liaison with the current study, would reinforce the possibility of the existence of " $\Lambda$ " shaped vortices that ex- 


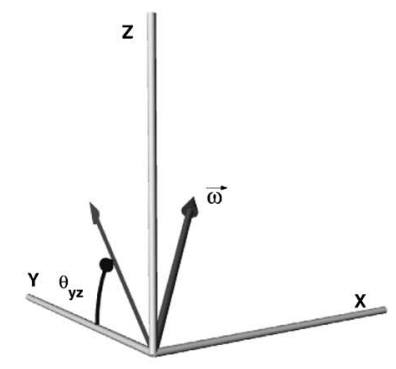

(a)

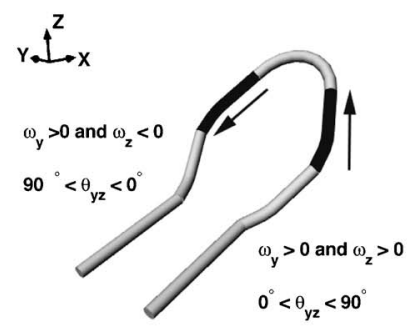

(c)

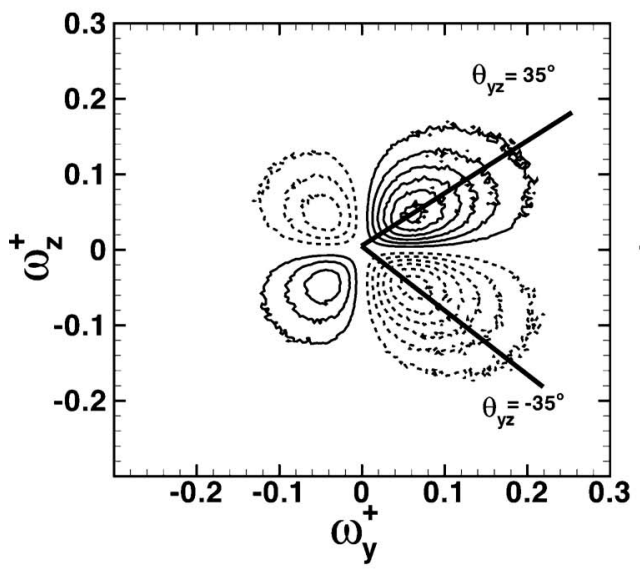

(e)

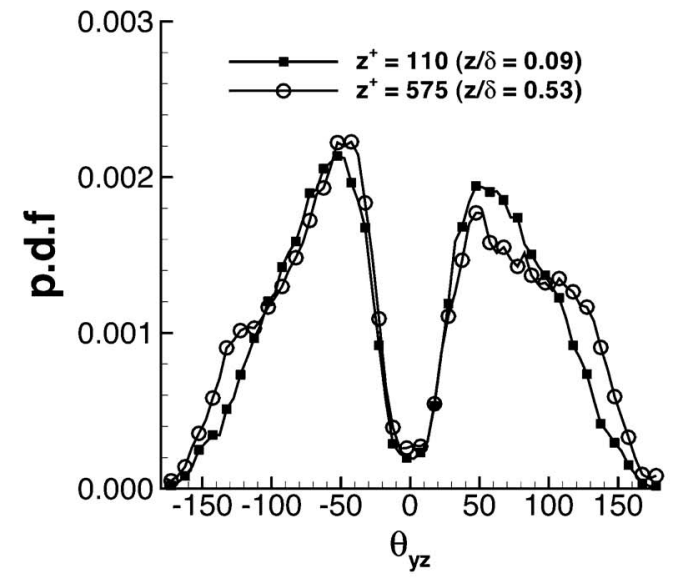

(b)

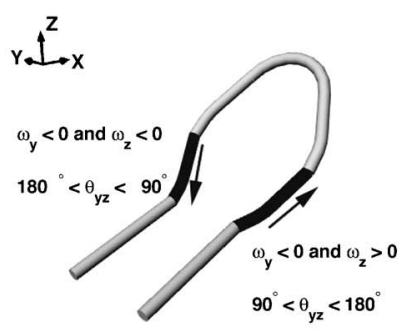

(d)

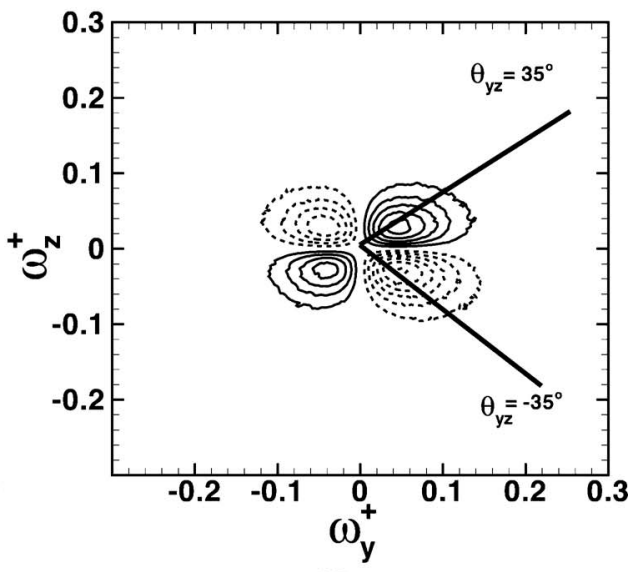

(f)
FIG. 13. (a) Schematic representation of $\theta_{y z}$, (b) pdf of $\theta_{y z}$, (c) and (d) schematic representations of a hairpin loop. Covariance of $\left(\omega_{y}, \omega_{z}\right)$ at (e) $z^{+}$ $=110$ and (f) $z / \delta=0.53$. The contour increment is \pm 0.01 and the first level shown is \pm 0.01 . Negative contours are shown with dotted lines and the zero contour is not shown. tend down to the wall. Although the second and third peaks are at $75^{\circ}$ and $-75^{\circ}$, the distribution surrounding the peaks indicates vortex cores with a range of orientations. For example, the pdf shows the presence of significant numbers of cores with $\theta_{y x}>90^{\circ}$ and $\theta_{y x}<-90^{\circ}$. These inclinations suggest the possibility of the presence of vortex rods tilted outwards [similar to the lower parts of the " $\Omega$ " shaped neck that are tilted away from each other as shown in Fig. 12(d)].

Figures 12(e) and 12(f) show covariance plots at $z^{+}$ $=110$ and $z / \delta=0.53$. The figures reveal the dominance of the contributions from quadrant $1\left(\omega_{x}>0, \omega_{y}>0\right)$ and quadrant $2\left(\omega_{x}<0, \omega_{y}>0\right)$, which is consistent with the distribution of $\theta_{y x}$ computed from individual vortex cores. The angles of inclination made with the positive $y$ axis can be inferred from the locations of the peaks of the covariances. The angles were determined to be $43^{\circ}$ and $-41^{\circ}$ at $z^{+}=110$ and $47^{\circ}$ and $-45^{\circ}$ at $z / \delta=0.53$ which represent smaller magnitudes than the peak locations in the $\theta_{y x}$ distribution. However, the covariance plots at both wall-normal locations seem to follow the general trend exhibited in $\theta_{y x}$ distributions and indicate a variety of both inward and outward tilted vortex rods (consistent with a range of " $\Lambda$ " and " $\Omega$ " shaped hairpin loops).

Figure 13(a) defines $\theta_{y z}$, which is the inclination angle made by the projection of the vorticity vector with the positive $y$ axis in the $y-z$ plane. Figure 13(b) shows the pdf of $\theta_{y z}$ at $z^{+}=110$ and $z / \delta=0.53$. This plot has peaks at $\pm 45^{\circ}$ (at both wall-normal locations) suggesting that most structures are inclined at $\pm 45^{\circ}$ in the $y-z$ plane. This result can be explained again by using the inward tilted " $\Lambda$ " shaped por- 


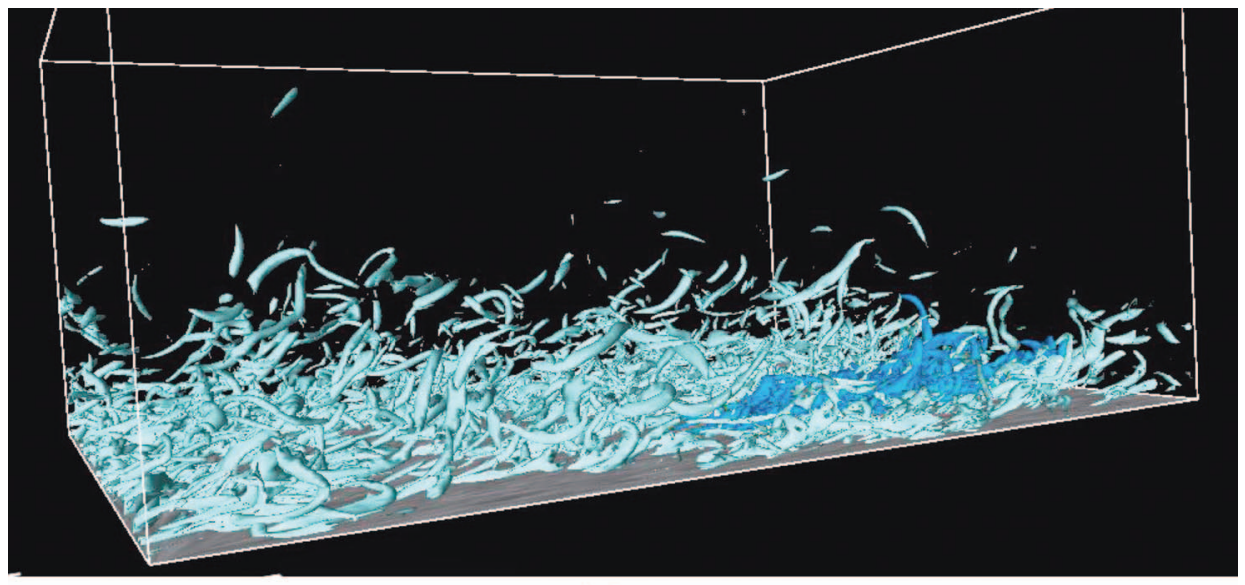

(a)

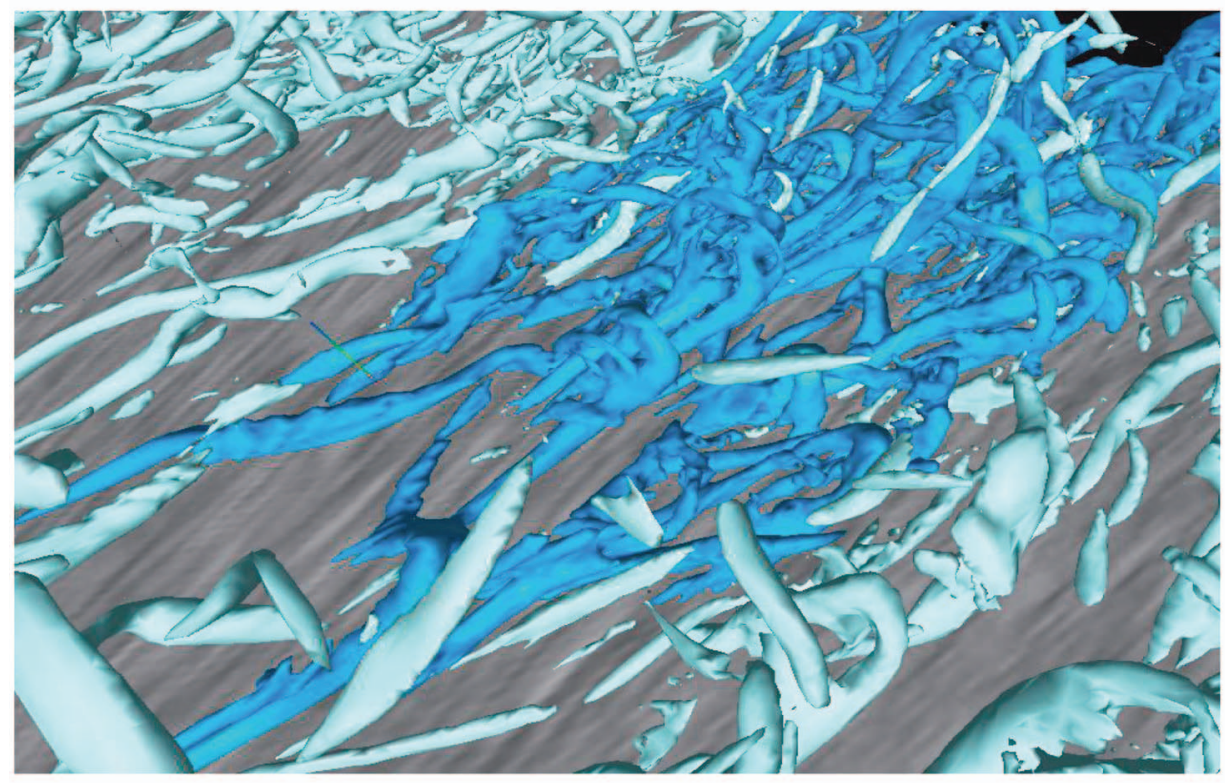

FIG. 14. (Color) (a) Perspective view of an isosurface plot of $\lambda_{3 \mathrm{D}}$ at an instant taken from a DNS of channel flow (Ref. 28). (b) Top view of a portion of (a). The visualization was performed at the Department of Computer Science, University of Minnesota.

(b)

tion of a hairpin loop shown in Fig. 13(c). All positive legs of a " $\Lambda$ " shaped vortex will have an inclination in the first quadrant $\left(0^{\circ}<\theta_{y z}<90^{\circ}\right)$ while the negative legs will have inclination in the fourth quadrant $\left(-90^{\circ}<\theta_{y z}<0^{\circ}\right)$. Note that the peaks are broad and include a wide range of eddy structures at various angles. The presence of cores with $\theta_{y z}>90^{\circ}$ and $\theta_{y z}<-90^{\circ}$ is consistent with the outward tilted lower portion of hairpin loops as shown in Fig. 13(d).

Figures 13(e) and 13(f) show the covariance of $\left(\omega_{y}, \omega_{z}\right)$ at $z^{+}=110$ and $z / \delta=0.53$, respectively. The covariance plots at $z^{+}=110$ reveal the dominance of the contributions from quadrant $1\left(\omega_{y}>0, \omega_{z}>0\right)$ and quadrant $4\left(\omega_{y}>0, \omega_{z}<0\right)$. The angles of inclination made with the positive $y$ axis can be inferred from the location of the peaks in these covariances in the first and fourth quadrants. The angles were determined to be $41^{\circ}$ and $-38^{\circ}$ at $z^{+}=110$ and $30^{\circ}$ and $-33^{\circ}$ at $z / \delta=0.53$ and are in accordance with the peaks found in the $\theta_{y z}$ distribution in Fig. 13(b).

\section{DISCUSSION}

The pdf of $\theta_{i}$ showed that the boundary layer is comprised mostly of forward-leaning cores; however a small number of cores lean against the flow. The presence of backward-leaning cores can be observed in visualizations of instantaneous fields from DNS datasets such as those of Ferrante et al. $^{29}$ in a turbulent boundary layer and Tanahashi et $a l^{30}$ in a channel flow. Another example is given in Fig. 14 which shows a three-dimensional isosurface plot of $\lambda_{3 \mathrm{D}}$ in an instantaneous field taken from a DNS of a channel flow. ${ }^{28}$ The figure shows that the channel contains structures with a wide range of inclination angles. The cores are predominantly forward leaning, although backward leaning cores can also be seen.

In the log region, the number of forward-leaning cores is much larger than the number of backward-leaning cores as seen from Fig. 11(b). The correlations in Fig. 4 indicate that forward-leaning cores are present over a range of scales with varying population density, consistent with the wall-wake model proposed by Perry and Marusic. ${ }^{31,32}$ The range of sizes for the backward-leaning cores is found to be smaller. A higher threshold on swirl strength value decreases the relative number density of backward leaning cores [as seen in Fig. 10(a)] suggesting that backward-leaning cores are on average weaker than their forward leaning counterparts. Also, the total number density of $\theta_{i}$ in Fig. 11(b) shows that 
the number of backward-leaning cores remains relatively constant between the log and wake regions. All of the above findings are consistent with the presence of small-scale backward-leaning cores whose number density and range of size are seemingly unchanged across the boundary layer. We would expect these smaller, weaker structures to have a relatively weak influence on boundary layer energetics, i.e., these weaker eddies likely make a much weaker contribution to the production than the larger, predominantly forwardleaning eddies. Similarly, we expect the stronger, larger forward-leaning eddies to distort and convect the weaker eddies by induction. It is likely that these stronger eddies induce a wide distribution of inclination angles within weaker eddies, so that their angle distribution becomes relatively isotropic.

The distributions of $\theta_{y x}$ and $\theta_{y z}$ point to the dominance of inward leaning vortex rods (consistent with the " $\Lambda$ " shaped neck of a hairpin loop) in the planes examined in this study. The overall distribution suggests that a variety of shapes cross the chosen measurement planes, ranging from " $\Lambda$ " shaped to " $\Omega$ " shaped vortices. The DNS visualization in Fig. 14 shows the presence of both " $\Lambda$ " and " $\Omega$ " shaped necks for the vortices, although the dominance of one group over the other is not clear.

The probability distributions of all the inclination and elevation angles presented in this paper were compared to those computed from a channel flow DNS dataset with similar Reynolds number ${ }^{28}$ and presented in Saikrishnan et al. ${ }^{33}$ The core identification technique was applied to both fully resolved DNS data and data "smoothed" to the PIV resolution. In general, the resulting resolved and smoothed DNS angle distributions were remarkably similar to those derived from the PIV data, thus validating the technique used to measure the gradients and lending further support to the conclusions of this study.

\section{CONCLUSIONS}

Simultaneous dual-plane PIV experiments were performed to compute all nine velocity gradients in a turbulent boundary layer at two wall-normal locations. The measurements were used to compute the complete vorticity vector in order to study the geometric orientations of vortex cores. Two-point correlations were calculated to identify the characteristic shapes of eddies and to compute the distribution of instantaneous vortex inclination angles at both wall-normal locations. The conclusions based on the correlations and the distribution of the instantaneous core angles are as follows:

(i) The pdf of the elevation angle $\left(\theta_{e}\right)$ reveals a most probable angle of $38^{\circ}$ at $z^{+}=110$ and $33^{\circ}$ at $z / \delta$ $=0.53$. This result, which portrays a decreasing trend in the angle with wall-normal location is consistent with the findings of Ong and Wallace. ${ }^{10}$ However, the distributions are broad indicating a wide range of inclination angles.

(ii) The pdf of the eddy inclination angle $\left(\theta_{i}\right.$, as projected onto the streamwise-wall-normal plane) has peaks at $46^{\circ}$ at $z^{+}=110$ and $42^{\circ}$ at $z / \delta=0.53$. This value compares favorably with other studies which indicated that average hairpin vortices are inclined at $45^{\circ}$ with the streamwise direction. The angle $\theta_{i}$ is also in accordance with the orientation of the principal strain axis in the boundary layer.

(iii) The distribution of $\theta_{y x}$ (angle made with the $y$ axis in the $x-y$ plane) contains broad modes consistent with the presence of inward leaning vortex rods (as would occur in a " $\Lambda$ " shaped hairpin loop) and spanwise oriented cores at both wall-normal locations. The pdf of the $\theta_{y z}$ (angle made with the $y$ axis in the $y-z$ plane) shows peaks at $\pm 45^{\circ}$ at both wall-normal locations that further reinforces the presence of inwardleaning vortex rods. The distributions of both $\theta_{y x}$ and $\theta_{y z}$ are broad, however, indicating a variety of structures ranging from an inward-leaning " $\Lambda$ " shape to an " $\Omega$ " shape containing outward leaning cores. It must be noted that the instantaneous cores identified are not necessarily joined as hairpins and that the proposed hairpin model is a simplified representative structure.

(iv) Two point autocorrelations of $\lambda_{1}, \lambda_{2}, \lambda_{3}$, and $\lambda_{4}$ (values of $\lambda_{3 \mathrm{D}}$ separated based on the quadrants of $\left.\omega_{x}-\omega_{z}\right)$ indicate that a representative forward-leaning vortex core is larger in scale than its backward-leaning counterpart. The shapes of the contours indicate an inward leaning " $\Lambda$ " shape for a representative eddy at these wall-normal locations.

(v) The number of forward-leaning cores decreased with increasing wall-normal distance, which is consistent with kinematic models based on the attached eddy hypothesis. ${ }^{31,34}$ Qualitatively, these features are also seen in visualizations from DNS studies of boundary layers and channel flows at similar Reynolds number. It is also noted that the absolute number density of the backward-leaning cores at $z^{+}=110$ and $z / \delta=0.53$ remained relatively constant suggesting a universality in the population of the backward leaning cores across the boundary layer. This suggests that these vortex cores are part of weaker (passive) structures that have perhaps been distorted and convected by the larger, predominantly forward-leaning eddies associated with the local shear.

\section{ACKNOWLEDGMENTS}

The authors express sincere thanks to Professor Victoria Interrante and Matt Heinzen for visualization of the channel flow dataset. The authors also thank Professor Robert Moser for providing the channel flow dataset. We are indebted to Dr. Nicholas Hutchins, William Hambleton, and Aizaz Bhuiyan for their help in data acquisition and many discussions during the course of this study. Financial support from the National Science Foundation through Grants No. ACI9982274, No. CTS-9983933, and No. CTS-0324898, the Graduate School of University of Minnesota, and the David and Lucile Packard Foundation is gratefully acknowledged.

${ }^{1}$ R. J. Adrian, C. D. Meinhart, and C. D. Tomkins, "Vortex organization in the outer region of the turbulent boundary layer," J. Fluid Mech. 422, 1 (2000). 
${ }^{2}$ T. Theodorsen, "Mechanism of turbulence," in Proceedings of the Second Midwestern Conference on Fluid Mechanics, March 17-19 (Ohio State University, Columbus, OH, 1952).

${ }^{3} \mathrm{G}$. R. Offen and S. J. Kline, "A proposed model of the bursting process in turbulent boundary layer," J. Fluid Mech. 70, 209 (1975).

${ }^{4}$ B. Ganapathisubramani, E. K. Longmire, and I. Marusic, "Characteristics of vortex packets in turbulent boundary layers," J. Fluid Mech. 478, 35 (2003).

${ }^{5}$ M. R. Head and P. Bandyopadhyay, "New aspects of turbulent boundarylayer structure," J. Fluid Mech. 107, 297 (1981).

${ }^{6} \mathrm{P}$. Moin and J. Kim, "The structure of the vorticity field in turbulent channel flow. Part 1. Analysis of instantaneous and statistical correlation," J. Fluid Mech. 155, 441 (1985).

${ }^{7}$ J. Kim and P. Moin, "The structure of the vorticity field in turbulent channel flow. Part 2. Study of ensemble-averaged fields," J. Fluid Mech. 162, 339 (1986)

${ }^{8}$ A. E. Alving, A. J. Smits, and J. H. Watmuff, "Turbulent boundary layer relaxation from convex curvature," J. Fluid Mech. 211, 529 (1990).

${ }^{9}$ I. Marusic, "On the role of large-scale structures in wall turbulence," Phys. Fluids 13, 735 (2001).

${ }^{10}$ L. Ong and J. M. Wallace, "Joint probability density analysis of the structure and dynamics of the vorticity field of a turbulent boundary layer," J. Fluid Mech. 367, 291 (1998).

${ }^{11}$ A. Honkan and Y. Andreopoulos, "Vorticity, strain-rate and dissipation characteristics in the near-wall region of turbulent boundary layers," J. Fluid Mech. 350, 29 (1997).

${ }^{12}$ J. Zhou, R. J. Adrian, S. Balachandar, and T. M. Kendall, "Mechanisms for generating coherent packets of hairpin vortices in channel flow," J. Fluid Mech. 387, 353 (1999).

${ }^{13}$ J. Jeong and F. Hussain, "On the identification of a vortex," J. Fluid Mech. 258, 69 (1995).

${ }^{14}$ M. S. Chong, A. E. Perry, and B. J. Cantwell, "A general classification of three-dimensional flow fields," Phys. Fluids A 2, 765 (1990).

${ }^{15}$ G. Haller, "An objective definition of a vortex," J. Fluid Mech. 525, 1 (2005).

${ }^{16}$ B. Ganapathisubramani, E. K. Longmire, I. Marusic, and S. Pothos, "Dual-plane PIV technique to measure complete velocity gradient tensor in a turbulent boundary layer," Exp. Fluids 39, 222 (2005).

${ }^{17}$ B. Ganapathisubramani, "Investigation of turbulent boundary layer structure using stereoscopic particle image velocimetry," Ph.D. thesis, University of Minnesota (2004).

${ }^{18}$ J.-L. Balint, J. M. Wallace, and P. Vukoslavcevic, "The velocity and vorticity vector fields of a turbulent boundary layer. Part 2. Statistical properties," J. Fluid Mech. 228, 53 (1991).

${ }^{19}$ J.-L. Balint, J. M. Wallace, and P. Vukoslavcevic, "A study of vortical structure of the turbulent boundary layer," in Advances in Turbulence, edited by G. Comte-Bellot and J. Mathieu (Springer, New York, 1987), pp. 456-464.

${ }^{20} \mathrm{G}$. C. Lemonis, "An experimental study of the vector fields of velocity and vorticity in turbulent flows," Ph.D. thesis, Swiss Federal Institute of Technology, Zurich, Institute of Hydromechanics and Water Resources (1995).

${ }^{21}$ P. R. Spalart, "Direct simulation of turbulent boundary layer up to $\operatorname{Re}_{\theta}$ =1410," J. Fluid Mech. 187, 61 (1988).

${ }^{22}$ B. Ganapathisubramani, N. Hutchins, W. T. Hambleton, E. K. Longmire, and I. Marusic, "Investigation of large-scale coherence in a turbulent boundary layer using two-point correlations," J. Fluid Mech. 524, 57 (2005).

${ }^{23}$ R. Cucitore, M. Quadrio, and A. Baron, "On the effectiveness and limitations of local criteria for the identification of a vortex," Eur. J. Mech. B/Fluids 18, 261 (1999).

${ }^{24}$ R. J. Adrian, K. T. Christensen, and Z. C. Liu, "Analysis and interpretation of instantaneous turbulent velocity fields," Exp. Fluids 29, 275 (2000).

${ }^{25}$ J. M. Wallace, H. Eckelmann, and R. S. Brodkey, "The wall region in turbulent shear flow," J. Fluid Mech. 54, 39 (1972).

${ }^{26} \mathrm{~W}$. W. Willmarth and S. S. Lu, "Structure of the Reynolds stress near the wall," J. Fluid Mech. 55, 65 (1972).

${ }^{27}$ J. M. Wallace and R. S. Brodkey, "Reynolds stress and joint probability density distributions of the $u-v$ plane of a turbulent channel flow," Phys. Fluids 20, 351 (1977)

${ }^{28}$ J. C. del Alamo, J. Jimenez, P. Zandonade, and R. D. Moser, "Scaling of the energy spectra of turbulent channels," J. Fluid Mech. 500, 135 (2004).

${ }^{29}$ A. Ferrante, S. Elghobashi, P. Adams, M. Valenciano, and D. Longmire, "Evolution of quasistreamwise vortex tubes and wall streaks in a bubbleladen turbulent boundary layer over a flat plate," Phys. Fluids 16, S2 (2004).

${ }^{30}$ M. Tanahashi, S. J. Kang, T. Miyamoto, S. Shiokawa, and T. Miyauchi, "Scaling law of fine scale eddies in turbulent channel flows up to $\operatorname{Re}_{\tau}$ =800," Int. J. Heat Mass Transfer 25, 331 (2004).

${ }^{31}$ A. E. Perry and I. Marusic, "A wall-wake model for the turbulence structure of boundary layers. Part 1. Extension of the attached eddy hypothesis," J. Fluid Mech. 298, 361 (1995).

${ }^{32}$ I. Marusic and A. E. Perry, "A wall-wake model for the turbulence structure of boundary layers. Part 2. Further experimental support," J. Fluid Mech. 298, 389 (1995).

${ }^{33}$ N. Saikrishnan, I. Marusic, and E. K. Longmire, "Assessment of dual plane PIV measurements in wall turbulence using DNS data," in Proceedings of the 6th International Symposium on Particle Image Velocimetry, 21-23 September 2005, Pasadena, CA.

${ }^{34}$ A. E. Perry and M. S. Chong, "On the mechanism of wall turbulence," J. Fluid Mech. 119, 173 (1982). 


\section{University Library}

\section{- M M I E E R VA A gateway to Melbourne's research publications}

Minerva Access is the Institutional Repository of The University of Melbourne

Author/s:

Ganapathisubramani, B.;Longmire, E. K.;Marusic, I.

Title:

Experimental investigation of vortex properties in a turbulent boundary layer

Date:

2006

Citation:

Ganapathisubramani, B., Longmire, E. K., \& Marusic, I. (2006). Experimental investigation of vortex properties in a turbulent boundary layer. Physics of Fluids, 18(055105), 1-14.

Publication Status:

Published

Persistent Link:

http://hdl.handle.net/11343/34776 